\title{
Reactions of 1,4-Dianion of Methyl 2-Thienyl Ketone N-Ethoxycarbonylhydrazone with Carboxylic Acid Derivatives and the Synthesis of Pyrazolotriazin-7-ones
}

\author{
Zuhal Turgut ${ }^{\star}$ and Nüket Öcal \\ Department of Chemistry; Faculty of Arts and Sciences, Yildiz Technical University, \\ Danutpasa Campus, 34010 Topkapi, Istanbul, Turkey \\ Received Jamain 11, 2002
}

Kevwords : Dianion reactions, 1,3,5-Trisubstituted pyrazoles, Pyrazolotriazin-7-ones.

Pyrazoles and pyrazolotriazines are important materials for medicinal, agricultural and other studies. ${ }^{1+4}$ Diverse biological activities such as bactericidal, pesticidal, anticonvulsant, tuberculostatic, anti-inflammatory, etc., have been found to be associated with pyrazole derivatives. In recent years, several new methods for the preparation of pyrazole derivatives have been reported in the literatures. ${ }^{5.7}$ However, little attention has been directed to obtain 3-hetero substituted pyrazoles. Thus, we reported here the synthesis of some 3 thiophen-2-yl-5-substituted pyrazoles by the reaction of butyl formate, methyl benzoate, ethyl benzoylacetate, methyl 3-chlorobenzoate, ethyl hexanoate, ethyl pyruvate, ethyl phenylglyoxylate, acetyl chloride and phenoxyacetyl chloride with dianion of methyl 2-thienyl ketone N-ethoxycarbonylhydrazone. Moreover, 1,4-dianion of $\mathrm{N}$-monosubstituted hydrazones $^{8}$ have received much attention as synthetic intermediates owing to their interesting reactivities and preparative significance.

In a typical reaction, a freshly prepared methyl 2-thienyl ketone N-ethoxycarbonylhydrazone 1 (mp. $114^{\circ} \mathrm{C}$ ) which was obtained from condensation of methyl 2-thienyl ketone and $\mathrm{N}$-ethoxycarbonylhydrazide ${ }^{9}$ in ethanol was dissolved in tetrahydrofuran and treated with 2 molar equiv, of $n$-buthyllithium in hexane under argon. To the resulting reddish brown colored solution 2 was added methyl 2-thienyl ketone Nethoxycarbonylhydrazone (1) one molar equivalent of esters or acid chlorides at $-78^{\circ} \mathrm{C}$, and this was followed by acid cyclization with $3 \mathrm{~N}$ hydrochloric acid, and chromatography of the product. Optimum yields of products were obtained when the ratio of hydrazone : base : ester (or acid chloride) was $1: 2: 1$; this is consistent with the proposed mechanism. The sequence in Scheme 1 would account for the results obtained. We also treated $\mathbf{3 f}$ and $\mathbf{3 g}$ with phenylhydrazine in methanol under reflux. The hydrazone indermediate was converted into the corresponding 4-methyl (or phenyl)-6-phenyl-2-(thiophen-2-yl)-6H-pyrazolo[1,5-d]$[1,2,4]$ triazin-7-ones $\mathbf{4 a}$, b by treatment with potassium hydroxide in methanol under reflux. ${ }^{10}$

$\mathrm{C}=\mathrm{O}$ band of ester group which is attached on nitrogen appeared at $1690-1670 \mathrm{~cm}^{-1}$. The methylene and methyl signals of ethyl group of ester appeared at $\delta$ 1.49-1.44 ppm as a quartet and $\delta 4.57-4.50 \mathrm{ppm}$ as a triplet in their ${ }^{1} \mathrm{H}$ NMR

"Corresponding author. E-mail: zturgut(ay yildiz.edu.tr

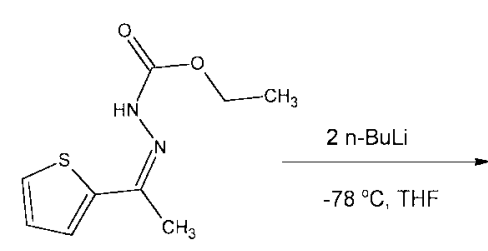

1

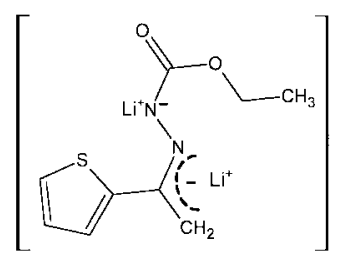

2
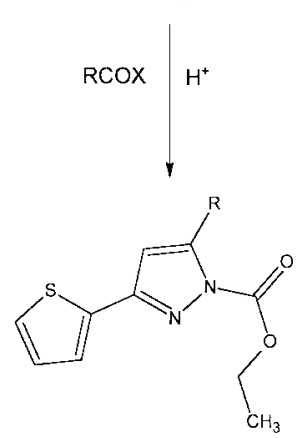

$3 \mathrm{a}-\mathrm{i}$

Scheme 1. Synthesis of 3a-i.

spectra. respectively in $\mathrm{CDCl}_{3}$. Compound 3f and 3h had methyl signals as a singlet at $2.25 \mathrm{ppm}$ and $2.43 \mathrm{ppm}$, respectively. Besides. methylene groups of the substituents (benzoylmethyl- and phenoxymethyl-) at 5-position of the pyrazole ring displayed a singlet at $\delta 4.52 \mathrm{ppm}$ for $3 \mathrm{c}$ and a singlet at $\delta 5.35$ ppm for $3 \mathbf{i}$. Compound 3 e gave multiplet peaks at $\delta 1.36-1.07 \mathrm{ppm}$ due to hexyl substituent. 4a,b are also in agreement with the structures reported."

The ${ }^{1{ }^{3} \mathrm{C}} \mathrm{NMR}$ spectra of the compounds $\mathbf{3 a}, \mathbf{b}, \mathbf{d}, \mathbf{e}, \mathbf{h , i}$ in $\mathrm{CDCl}_{3}$ gave $\mathrm{C}=\mathrm{O}$ signals at $150.00-139.53 \mathrm{ppm}$. On the other hand, phenyl substituted $\mathrm{C}=\mathrm{O}$ signal appeared for $3 \mathrm{c}$ at $\delta 201.60 \mathrm{ppm}$ for $3 \mathrm{f}$ at $191.12 \mathrm{ppm}$, for $\mathbf{3 g}$ at $183.49 \mathrm{ppm}$ respectively. The structures of the all compounds have also been confirmed by their mass spectral data.

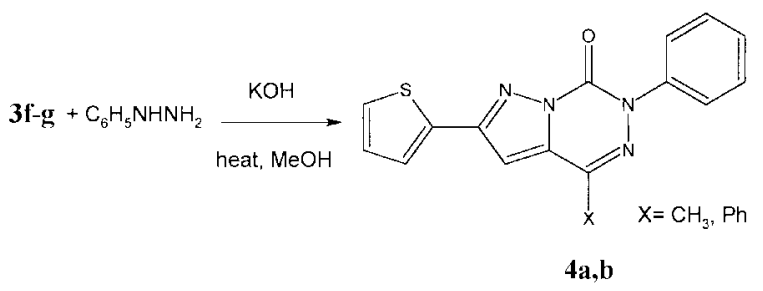

Scheme 2. Synthesis of $\mathbf{4 a - b}$. 
Table 1. Yields of $\mathbf{3 a - i}$

\begin{tabular}{cllc}
\hline Compound & \multicolumn{1}{c}{$\mathrm{R}$} & \multicolumn{1}{c}{$\mathrm{X}$} & Yield $\%)$ \\
\hline $\mathbf{3 a}$ & $\mathrm{H}$ & $\mathrm{OCH}_{2}\left(\mathrm{CH}_{2}\right)_{2} \mathrm{CH}_{3}$ & 78 \\
$\mathbf{3 b}$ & $\mathrm{C}_{6} \mathrm{H}_{5}$ & $\mathrm{OCH}_{3}$ & 92 \\
$\mathbf{3 c}$ & $\mathrm{C}_{6} \mathrm{H}_{5} \mathrm{COCH}_{2}$ & $\mathrm{OC}_{2} \mathrm{H}_{5}$ & 53 \\
$\mathbf{3 d}$ & $m-\mathrm{Cl} \mathrm{C}_{6} \mathrm{H}_{5}$ & $\mathrm{OCH}_{3}$ & 58 \\
$\mathbf{3 e}$ & $\mathrm{CH}_{3}\left(\mathrm{CH}_{2}\right)_{4} \mathrm{CH}_{2}$ & $\mathrm{OC}_{2} \mathrm{H}_{5}$ & 72 \\
$\mathbf{3 f}$ & $\mathrm{CH}_{3} \mathrm{CO}$ & $\mathrm{C}_{6} \mathrm{H}_{5}$ & 44 \\
$3 \mathbf{3 g}$ & $\mathrm{C}_{6} \mathrm{H}_{5} \mathrm{CO}$ & $\mathrm{CO}_{6} \mathrm{H}_{5}$ & 56 \\
$\mathbf{3 h}$ & $\mathrm{CH}_{3}$ & $\mathrm{Cl}$ & 23 \\
$3 \mathbf{3 i}$ & $\mathrm{C}_{6} \mathrm{H}_{5} \mathrm{OCH}$ & $\mathrm{Cl}$ & 43 \\
\hline
\end{tabular}

\section{Experimental Section}

Melting points are uncorrected and were measured in open capillaries with an Electrothermal LA 9100 melting point apparatus. Infrared spectra were recorded on a Philips PU 9714 spectrometer as $\mathrm{KBr}$ pellets unless otherwise indicated. NMR spectra were determined on a Nicolet $300 \mathrm{MHz}$ (compounds 3a, e-h, $\mathbf{4 a}, \mathbf{b}$ ) and on a Varian $200 \mathrm{MHz}$ spectrometers in $\mathrm{CDCl}_{3}$ with TMS as internal standard. Mass spectra of new compounds were obtained with a Shimadzu GS/MS QP 2000A spectrometer with $70 \mathrm{ev}$ electron impact ionization. Column chromatography was performed with silica gel $60(70-230$ mesh $)$ purchased from E. Merck AG. Thin layer choromatography (TLC) were carried out using Merck 5554 and Watmann 4410222 silica gel sheets with fluorescent indicators.

Synthesis of 5-substituted 1-ethoxycarbonyl-3-(2-thienyl) pyrazoles (3a-i); General Procedure. To a THF solution of $1(2.42 \mathrm{mmol})$ was added a hexane solution of $n$-buthyllithium $(5.32 \mathrm{mmol})$ with stirring at $-78^{\circ} \mathrm{C}$ under argon. To the resulting reddish brown colored solution was added dropwise a THF solution of an ester or acid chloride $(2.18$ mmol). After complete fading of the color, the reaction mixture 2 was stirred for additional 2 hours at $-78^{\circ} \mathrm{C}$, and the solvent was removed in vacuo. The residual mixture was treated with conc. $\mathrm{HCl}$, acetic acid, water and methanol (10 $\mathrm{ml}$ each), and stirred for 2 hours at room temperature. After methanol was evaporated, the residue was extracted with ether, and the extract chromatographed on silica gel column with toluene. Evaporation of the solvent gave pure compound 3 .

3a: mp. $129^{\circ} \mathrm{C}$ : IR (KBr): $v 1670 \mathrm{~cm}^{-1}:{ }^{1} \mathrm{H} \mathrm{NMR}\left(\mathrm{CDCl}_{3}\right.$, $300 \mathrm{MHz}): \delta 8.14-6.62(\mathrm{~m}, 5 \mathrm{H}$, aromatic), $4.56-4.50(\mathrm{q}, 2 \mathrm{H}$, $\left.\mathrm{OCH}_{2} \mathrm{CH}_{3}\right), 1.49-1.45\left(\mathrm{t}, 3 \mathrm{H}, \mathrm{OCH}_{2} \mathrm{CH}_{3}\right) \mathrm{ppm}:{ }^{13} \mathrm{C} \mathrm{NMR}$ $\left(\mathrm{CDCl}_{3}, 300 \mathrm{MHz}\right): \delta 151.88-107.54$ (aromatic), $149.97(\mathrm{~N}-$ $\mathrm{C}=\mathrm{O}), 65.31\left(\mathrm{OCH}_{2}\right), 14.88\left(\mathrm{CH}_{3}\right)$ ppm: MS: m/z $222.26\left(\mathrm{M}^{-}\right)$, $\left(\mathrm{C}_{10} \mathrm{H}_{10} \mathrm{~N}_{2} \mathrm{O}_{2} \mathrm{~S}\right)$.

3b: mp. $114^{\circ} \mathrm{C}$ : IR (KBr): $v 1680 \mathrm{~cm}^{-1}:{ }^{1} \mathrm{H} \mathrm{NMR}\left(\mathrm{CDCl}_{3}\right.$, $200 \mathrm{MHz}): \delta 7.65-6.52(\mathrm{~m}, 9 \mathrm{H}$, aromatic), $4.55-4.51(\mathrm{q}, 2 \mathrm{H}$, $\left.\mathrm{OCH}_{2} \mathrm{CH}_{3}\right), 1.48-1.44\left(\mathrm{t}, 3 \mathrm{H}, \mathrm{OCH}_{2} \mathrm{CH}_{3}\right) \mathrm{ppm} ;{ }^{13} \mathrm{C} \mathrm{NMR}$ ( $\mathrm{CDCl}_{3}, 200 \mathrm{MHz}$ ): $\delta 158.97-105.37$ (aromatic), $141.31(\mathrm{~N}-$ $\mathrm{C}=\mathrm{O}), 62.80\left(\mathrm{OCH}_{2}\right), 13.90\left(\mathrm{CH}_{3}\right)$ ppm: MS: $\mathrm{m} / \mathrm{z} 298.35$ $\left(\mathrm{M}^{+}\right),\left(\mathrm{C}_{16} \mathrm{H}_{14} \mathrm{~N}_{2} \mathrm{O}_{2} \mathrm{~S}\right)$.

3c: mp. $136{ }^{\circ} \mathrm{C}$; IR (KBr): v $1750,1690 \mathrm{~cm}^{-1}:{ }^{1} \mathrm{H}$ NMR $\left(\mathrm{CDCl}_{3}, 200 \mathrm{MHz}\right): \delta 7.72-7.05(\mathrm{~m}, 9 \mathrm{H}$, aromatic), 4.54- $4.50\left(\mathrm{q}, 2 \mathrm{H}, \mathrm{OCH}_{2} \mathrm{CH}_{3}\right), 4.52\left(\mathrm{~s}, 2 \mathrm{H}, \mathrm{CH}_{2}\right), 1.46-1.45$ (t, $3 \mathrm{H}$, $\left.\mathrm{OCH}_{2} \mathrm{CH}_{3}\right)$ ppm; ${ }^{13} \mathrm{C}$ NMR $\left(\mathrm{CDCl}_{3}, 200 \mathrm{MHz}\right): \delta 201.60$ $(\mathrm{C}=\mathrm{O}), 155.38-118.17$ (aromatic), $139.53(\mathrm{~N}-\mathrm{C}=\mathrm{O}), 62.80$ $\left(\mathrm{OCH}_{2}\right), 38.41\left(\mathrm{CH}_{2}\right), 13.90\left(\mathrm{CH}_{3}\right)$ ppm; $\mathrm{MS}: \mathrm{m} / \mathrm{z} 340.39\left(\mathrm{M}^{+}\right)$, $\left(\mathrm{C}_{18} \mathrm{H}_{16} \mathrm{~N}_{2} \mathrm{O}_{2} \mathrm{~S}\right)$.

3d: mp. $108^{\circ} \mathrm{C}$; IR (KBr): $v 1680 \mathrm{~cm}^{-1}$; ${ }^{1} \mathrm{H}$ NMR (CDCl 3 , $200 \mathrm{MHz}): \delta 7.88-6.86(\mathrm{~m}, 8 \mathrm{H}$, aromatic), 4.57-4.53 (c, $2 \mathrm{H}$, $\mathrm{OCH}_{2} \mathrm{CH}_{3}$ ), $1.48-1.46$ (t, $3 \mathrm{H}, \mathrm{OCH}_{2} \mathrm{CH}_{3}$ ) ppm; ${ }^{13} \mathrm{C}$ NMR ( $\left.\mathrm{CDCl}_{3}, 200 \mathrm{MHz}\right): \delta 158.97-105.37$ (aromatic), 141.31 (N$\mathrm{C}=\mathrm{O}), 62.81\left(\mathrm{OCH}_{2}\right), 13.90\left(\mathrm{CH}_{3}\right) \mathrm{ppm} ; \mathrm{MS}: \mathrm{m} / \mathrm{z} 332.80$ $\left(\mathrm{M}^{-}\right),\left(\mathrm{C}_{16} \mathrm{H}_{13} \mathrm{~N}_{2} \mathrm{O}_{2} \mathrm{SCl}\right)$.

3e: mp. $138^{\circ} \mathrm{C} ; \mathrm{RR}(\mathrm{KBr}): v 1670 \mathrm{~cm}^{-1}$; ${ }^{1} \mathrm{HNMR}$ (CDCla, 300 $\mathrm{MHz}): \delta 7.72-6.38(\mathrm{~m}, 4 \mathrm{H}$, aromatic), 4.57-4.51 (c, $2 \mathrm{H}$, $\left.\mathrm{OCH}_{2} \mathrm{CH}_{3}\right), 2.97-2.95\left(\mathrm{t}, 2 \mathrm{H}, \mathrm{CH}_{2}\right), 1.73-1.67$ (p, 2H, $\mathrm{CH}_{2}$ ), 1.48-1.44 (t. $\left.3 \mathrm{H}, \mathrm{OCH}_{2} \mathrm{CH}_{3}\right), 1.36-1.07\left(\mathrm{~m}, 9 \mathrm{H}, \mathrm{CH}_{2}\right)$ ppm; ${ }^{13} \mathrm{C}$ NMR (CDCla, $300 \mathrm{MHz}$ ): $\delta 160.40-109.33$ (aromatic), $141.51(\mathrm{~N}-\mathrm{C}=\mathrm{O}), 62.80\left(\mathrm{OCH}_{2}\right), 31.46-27.37\left(\mathrm{CH}_{2}\right), 14.01$ $\left(\mathrm{CH}_{3}\right), \quad 13.89 \quad\left(\mathrm{CH}_{3}\right)$ ppm; $\mathrm{MS}: \mathrm{m}^{2} \mathrm{z} \quad 306.42 \quad\left(\mathrm{M}^{-}\right)$, $\left(\mathrm{C}_{16} \mathrm{H}_{22} \mathrm{~N}_{2} \mathrm{O}_{2} \mathrm{~S}\right)$.

3f: mp. $98{ }^{\circ} \mathrm{C}$ : IR (KBr): $v 1720,1670 \mathrm{~cm}^{-1}$; ${ }^{1} \mathrm{H}$ NMR $\left(\mathrm{CDCl}_{3}, 300 \mathrm{MHz}\right): \delta 7.52-7.22(\mathrm{~m}, 4 \mathrm{H}$, aromatic), 4.594.53 (q, $\left.2 \mathrm{H}, \mathrm{OCH}_{2} \mathrm{CH}_{3}\right), 2.25\left(\mathrm{~s}, 3 \mathrm{H}, \mathrm{CH}_{3}\right), 1.45-1.43$ (t, $3 \mathrm{H}$, $\left.\mathrm{OCH}_{2} \mathrm{CH}_{3}\right) ;{ }^{13} \mathrm{C}$ NMR $\left(\mathrm{CDCl}_{3}, 300 \mathrm{MHz}\right): \delta 191.12(\mathrm{C}=\mathrm{O})$, 156.31-112.55 (aromatic), $143.16(\mathrm{~N}-\mathrm{C}=\mathrm{O}), 63.43\left(\mathrm{OCH}_{2}\right)$, $12.75\left(\mathrm{CH}_{3}\right)$, ppm; MS: $264.30 \mathrm{~m} / \mathrm{z}\left(\mathrm{M}^{-}\right),\left(\mathrm{C}_{12} \mathrm{H}_{12} \mathrm{~N}_{2} \mathrm{O}_{3} \mathrm{~S}\right)$.

3g: mp. $107^{\circ} \mathrm{C}$; IR (KBr): $v 1710,1690 \mathrm{~cm}^{-1}$; ${ }^{1} \mathrm{H}$ NMR $\left(\mathrm{CDCl}_{3}, 300 \mathrm{MHz}\right): \delta 8.27-7.04(\mathrm{~m}, 9 \mathrm{H}$, aromatic), 4.54$4.51\left(\mathrm{q}, 2 \mathrm{H}, \mathrm{OCH}_{2} \mathrm{CH}_{3}\right), 1.48-1.42\left(\mathrm{t}, 3 \mathrm{H}, \mathrm{OCH}_{2} \mathrm{CH}_{3}\right) ;{ }^{13} \mathrm{C}$ NMR $\left(\mathrm{CDCl}_{3}, 300 \mathrm{MHz}\right) ; \delta 183.49(\mathrm{C}=\mathrm{O}), 157.41-110.30$ (aromatic), $140.71(\mathrm{~N}-\mathrm{C}=\mathrm{O}), 62.81\left(\mathrm{OCH}_{2}\right), 13.93\left(\mathrm{CH}_{3}\right)$, ppm: MS: $325.36 \mathrm{~m} / \mathrm{Z}\left(\mathrm{M}^{+}\right),\left(\mathrm{C}_{17} \mathrm{H}_{13} \mathrm{~N}_{2} \mathrm{O}_{3} \mathrm{~S}\right)$.

3h: mp. $126^{\circ} \mathrm{C}$ : IR (KBr): $v 1690 \mathrm{~cm}^{-1}$; ${ }^{l} \mathrm{H} \mathrm{NMR}\left(\mathrm{CDCl}_{3}\right.$, $300 \mathrm{MHz}): \delta 7.72-6.49(\mathrm{~m}, 4 \mathrm{H}$, aromatic), $4.57-4.51(\mathrm{q}, 2 \mathrm{H}$, $\left.\mathrm{OCH}_{2} \mathrm{CH}_{3}\right), 2.43\left(\mathrm{CH}_{3}\right), 1.46-1.43$ (t. $\left.3 \mathrm{H}, \mathrm{OCH}_{2} \mathrm{CH}_{3}\right) \mathrm{ppm}$; ${ }^{13} \mathrm{C}$ NMR ( $\left.\mathrm{CDCl}_{3}, 300 \mathrm{MHz}\right): \delta$ 159.58-105.69 (aromatic), $141.67(\mathrm{~N}-\mathrm{C}=\mathrm{O}), 62.80\left(\mathrm{OCH}_{2}\right), 13.90\left(\mathrm{CH}_{3}\right), 13.04\left(\mathrm{CH}_{3}\right)$ ppm: MS: $m / z 236.29\left(\mathrm{M}^{+}\right),\left(\mathrm{C}_{11} \mathrm{H}_{12} \mathrm{~N}_{2} \mathrm{O}_{2} \mathrm{~S}\right)$.

3i: mp. $139^{\circ} \mathrm{C}$; IR (KBr): $v 1685 \mathrm{~cm}^{-1}$ : ${ }^{l} \mathrm{H}$ NMR $\left(\mathrm{CDCl}_{3}\right.$, $200 \mathrm{MHz}): \delta 7.72-6.72\left(\mathrm{~m}, 9 \mathrm{H}\right.$, aromatic), $5.35\left(\mathrm{~s}, 2 \mathrm{H}, \mathrm{CH}_{2}\right)$, 4.57-4.51 (c, $\left.2 \mathrm{H}, \mathrm{OCH}_{2} \mathrm{CH}_{3}\right), 1.48-1.44\left(\mathrm{t}, 3 \mathrm{H}, \mathrm{OCH}_{2} \mathrm{CH}_{3}\right)$ ppm; ${ }^{13} \mathrm{C} \mathrm{NMR}\left(\mathrm{CDCl}_{3}, 200 \mathrm{MHz}\right): \delta 160.50-104.07$ (aromatic), $139.30(\mathrm{~N}-\mathrm{C}=\mathrm{O}), 64.80\left(\mathrm{OCH}_{2}\right), 62.95\left(\mathrm{OCH}_{2}\right), 14.25\left(\mathrm{CH}_{3}\right)$ ppm; MS: $\mathrm{m} / \mathrm{z} 328.39\left(\mathrm{M}^{+}\right),\left(\mathrm{C}_{17} \mathrm{H}_{16} \mathrm{~N}_{2} \mathrm{O}_{3} \mathrm{~S}\right)$.

General procedure for the synthesis of 4-substituted 6phenyl-2-thiophen-2-yl-6H-pyrazolo[1,5-d][1,2,4]triazin-7ones $(4 a, b)$. To a stirred solution of $3 f$ or $3 g(1 \mathrm{mmol})$ in methanol $(10 \mathrm{~mL})$ was added the phenylhydrazine (1 mmol) in methanol $(10 \mathrm{~mL})$ and the reaction was allowed to react at room temperature for $4 \mathrm{~h}$ under magnetic stirring. The solvent was evaporated under reduced pressure and the crude reaction product was purified by column chromatograply (cyclohexane-ethyl acetate $2: 1$ ) affording the pure compounds 4a, b.

4a: mp. $162^{\circ} \mathrm{C}$; IR (KBr): $v 1685 \mathrm{~cm}^{-1}$; ${ }^{1} \mathrm{H}$ NMR (CDCl${ }_{3}$, $300 \mathrm{MHz}): \delta 8.92-6.36(\mathrm{~m}, 9 \mathrm{H}$, aromatics), $2.48(\mathrm{~s}, 3 \mathrm{H}$, $\left.\mathrm{CH}_{3}\right) \mathrm{ppm} ;{ }^{13} \mathrm{C} \mathrm{NMR}\left(\mathrm{CDCl}_{3}, 300 \mathrm{MHz}\right): \delta 158.25-105.42$ (aromatics and carbonyl), $12.77\left(\mathrm{CH}_{3}\right) \mathrm{ppm} ; \mathrm{MS}: \mathrm{m} / \mathrm{z}$ 
$308.36\left(\mathrm{M}^{-}\right),\left(\mathrm{C}_{16} \mathrm{H}_{12} \mathrm{~N}_{4} \mathrm{OS}\right)$

4b: $\mathrm{mp} .132^{\circ} \mathrm{C}$ : IR (KBr): $v 1678 \mathrm{~cm}^{-1}$ : ${ }^{1} \mathrm{H}$ NMR $\left(\mathrm{CDCl}_{3}\right.$, $300 \mathrm{MHz}): \delta 8.87-6.43\left(\mathrm{~m}, 14 \mathrm{H}\right.$, aromatic) $\mathrm{ppm} ;{ }^{13} \mathrm{C} \mathrm{NMR}$ ( $\mathrm{CDCl}_{3}, 300 \mathrm{MHz}$ ): $\delta$ 156.13-109.42 (aromatics and carbonyl) ppm; MS: $\mathrm{m} / \mathrm{z} 370.42\left(\mathrm{M}^{-}\right),\left(\mathrm{C}_{21} \mathrm{H}_{14} \mathrm{~N}_{4} \mathrm{OS}\right)$.

Acknowledgment. This work was completed Dr. Ihsan Erdens laboratory in San Francisco State University. We thank to him for kindly helping for our work and NMR and mass measurements. We are grateful for a NATO postdoctoral fellowship, administered by Tubitak of Turkey to Dr. Öcal.

\section{References}

1. Hamaguchi. H.; Hino, T.; Kohno, E.; Andoh. N.; Nishinatsu, T.; Kodama, H. Eur: Pat. Appl. EP 1991, 395, 422: Chem. Abstr. 1991, $114,247265 \mathrm{q}$.
2. Hlasta. J. D.; Casey, B. F.; Ferguson. E. W.; Gangel, J. S.; Heimantı. R. M.; Jaeger, E. P.; Kullnig. K. R.; Gordon. J. R. . . Med. Chem. 1991. 34(5), 1560.

3. Shaban, M. A.; Abdou, N. A.: El-Meligie, S.: Taher, A. J. Pham. Sci. 1990, $4(1), 93$.

4. Shaban, M. A.; Abdou, N. A.; El-Meligie, S.; Taher, A. Bull. Fac. Pham. 1990. 28(3), 19.

5. Duncan. D. C,; Trumbo. T. A.; Almquist. C. D.; Lentz, T. A.; Beam, C. F. J. Heterocyllic Chem, 1987. 24, 555.

6. Beam, C. F.; Reames, D. C.; Hanris, C. E.; Dasher, L. W.: Hollinger, W. M.; Shealy, N. L.; Sandifer, R. M. J. Org. Chem. $1975,40,514$

7. Baddar, F. G.; Al-Hajjar, F. H.; El-Rayyes, N. R. J. Heterocycl. Chem. 1976. 13. 257.

8. Vedejs. E.; Stolle. W. T. Tetrahedron Lett. 1977. 135.

9. Diels, O. Ber: 1914, 47, 2183.

10. Lancelot. J. C.; Maume, D.; Robba, M. J. Heterocicl. Chem. $1981,18(7), 1319$.

11. Orazio. A.; Filippone, P.; Fiorucci. C.; Mantellini, F. Tetrahedron Letters 1999, 40.3891. 